\title{
OXIMETRIA DE PULSO: PRINCÍPIOS DE FUNCIONAMENTO E APLICAÇÕES
}

\author{
Adrielle Martins Monteiro Alves ${ }^{1}$ \\ Lílian Melo de Miranda Fortaleza \\ Lízia Daniela e Silva Nascimento ${ }^{3}$ \\ Diego Rodrigues Pessoa ${ }^{4}$ \\ Raimundo Barros de Araújo Júnior ${ }^{5}$
}

\begin{abstract}
Resumo: A monitorização padrão em unidades de terapia intensiva, centros cirúrgicos, áreas de recuperação, ambulâncias, dentre outros ocorre por meio da oximetria de pulso, onde os oxímetros de pulso são utilizados na determinação do nível de saturação de oxigênio (SpO2) no sangue arterial. Essa quantificação é feita através de sensores ópticos posicionados externamente ao paciente. O princípio da oximetria de pulso fundamenta-se na absorção da luz de comprimento de onda conhecido (660 e $940 \mathrm{~nm}$ ) pela hemoglobina oxigenada. Este trabalho trata-se de uma revisão integrativa e bibliográfica de publicações científicas no período entre 2006 e 2016, além de livros, dissertação de mestrado, tese de doutorado e manuais técnicos. O conhecimento dos princípios básicos de funcionamento da oximetria é importante porque permite o reconhecimento de leituras errôneas e suas limitações. A oximetria é capaz de distinguir hemoglobina oxigenada da desoxigenada, constituindo um método não invasivo, de fácil utilização, responsável por captar a saturação arterial de forma contínua e segura.
\end{abstract}

Palavras-chave: Oximetria; Sensor; Sinais; Perfusão Tecidual.

\footnotetext{
${ }^{1}$ Mestranda em Engenharia Biomédica/Universidade do Vale do Paraíba- UNIVAP, Brasil. E-mail: adriellemonteiro@hotmail.com.

${ }^{2}$ Doutoranda em Engenharia Biomédica/ Universidade do Vale do Paraíba- UNIVAP/ IP\&D - Instituto de Pesquisa e Desenvolvimento, Brasil. E-mail: Immfortaleza@yahoo.com.br.

${ }^{3}$ Doutoranda em Engenharia Biomédica/ Universidade do Vale do Parába- UNIVAPI IP\&D - Instituto de Pesquisa e Desenvolvimento, Brasil. E-mail: liziadaniela@hotmail.com.

${ }^{4}$ Mestrando em Engenharia Biomédica/ Universidade do Vale do Paraíba- UNIVAP/ IP\&D - Instituto de Pesquisa e Desenvolvimento, Brasil. E-mail: fisio.diegorodrigues@gmail.com.

${ }^{5}$ Doutorando em Engenharia Biomédica / Universidade do Vale do Paraíba- UNIVAP/ IP\&D - Instituto de Pesquisa e Desenvolvimento, Brasil. E-mail: rbarrosaraujojr@gmail.com.
} 